\title{
Immunohistochemical Expression of "HE4" in Endometrial Hyperplasia versus Endometrial Endometrioid Carcinoma
}

\author{
Amany Talaat Abd El-Hamed*, Samira Abd-Allah Mahmoud, Ahmed A. Soliman, Dina F. El-Yasergy \\ Department of Pathology, Faculty of Medicine, Cairo University, Cairo, Egypt
}

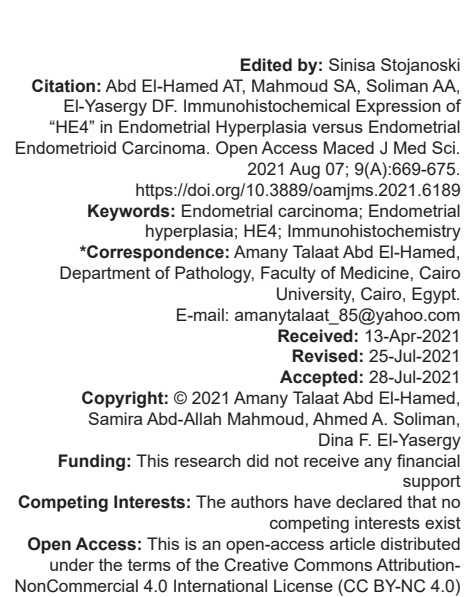

Introduction

Endometrial cancer is a malignant cancer arising from the endometrial epithelium, accounting for $20 \%$ to $30 \%$ of malignant tumors in the female reproductive system. Due to increased obesity, hypertension, diabetes, and prolonged life expectancy, the incidence and mortality of endometrial cancer have risen, with a tendency for onset at a younger age [1].

Approximately $90 \%$ of cases of endometrial cancer are sporadic, whereas the remaining $10 \%$ of cases are hereditary. Two major types are distinguished. Type I (estrogen-dependent) endometrial cancers represent the majority of sporadic cases, accounting for $80-85 \%$ of cases and these tumors are of endometrial histology. Type II is not estrogen-dependent, make up the remaining $10-15 \%$ of cases [2].

HE4, also known as whey acidic protein (WFDC2) was discovered in the human epididymal epithelium. It is a protease inhibitor which is associated with innate immunity and sperm maturation. HE4 is mainly expressed in germinal epithelium, oviduct epithelium, Bartholin's gland of females, and endometrial glands [3], [4]. Its overexpression is first observed in ovarian cancer tissue [5]. It has a better sensitivity, specificity than CA-125 in the diagnosis of ovarian cancer [6]. Subsequent research has shown that the overexpression of HE4 not only exists in patients with ovarian cancer but has also been observed in patients with endometrial carcinoma [7], [8].

CA125 is the major marker in the diagnosis and monitoring of endometrial cancer, but it is less sensitive and specific in diagnosing endometrial cancer compared to ovarian cancer; thus, it is only suitable for cases with advanced or recurrent endometrial cancer. Therefore, finding out other tumor markers is of great significant [9]. HE4 immunohistochemical expression in patients with endometrial carcinoma was significantly higher than that in patients with 
hyperplasia and in those with normal endometrial tissue, which provides a preliminary theoretical reference for basic research on the role of $\mathrm{HE} 4$ in endometrial cancer development [10], [11], [12]. The exact function of the HE4 protein is unknown, but some studies have demonstrated that overexpression of HE4 enhances cell adhesion and migration while suppression of HE4 markedly inhibits the growth of tumor cell [13]. Overexpression of HE4 in endometrial carcinoma cell lines induced proliferation of cancer cells in vitro and in vivo, supporting a function for HE4 in tumor progression. HE4 may be beneficial as a useful prognostic biomarker for endometrial carcinoma. Overexpression of HE4 is associated with increases in the FIGO stage and grade [14].

\section{Materials and Methods}

\section{Retrieval of cases}

This study included forty cases of endometrial endometrioid carcinoma and thirty cases of endometrial hyperplasia obtained through collection of archived paraffin blocks during the period from January 2018 till February 2019, from the Pathology Department, Kasr AL-Ainy, Faculty of Medicine, Cairo University. The forty cases of endometrial carcinoma were obtained from total hysterectomy with bilateral salpingoophrectomy specimens and the thirty cases of endometrial hyperplasia were obtained from endometrial biopsies.

The data collected from the pathology reports of these cases included age at the time of diagnosis, the extent of myometrial invasion, presence of tumor involvement of the cervix, adnexa, and parametrium.

Cases received neoadjuvant therapy or cases with missed data were excluded from the study.

\section{Histopathological examination}

Each paraffin block was re-cut by rotatory microtome at $4 \mu \mathrm{m}$ thickness then mounted on glass slides and stained by hematoxylin and eosin ( $\mathrm{H}$ and $\mathrm{E})$ for routine histopathological examination which included:

- $\quad$ Histological classification according to the latest World Health Organization recommendations [15].

- Histological grading according to the FIGO grading system [16].

- $\quad$ Pathological staging according to the FIGO staging system [17] and the eighth edition (2017) of the American Joint Committee on Cancer's AJCC Cancer Staging Manual [18].

\section{evaluation}

\section{HE4 Immunohistochemical staining and}

Paraffin sections were cut at $4 \mu \mathrm{m}$ thickness on positively charged slides. Immunostaining was done using BenchMark XT (Ventana) autostainer.

A section of epididymis was used as positive control according to the manufacturer recommendations.

HE4 Immunohistochemical staining results, brown-stained granules on the cell membrane and cytoplasm, were regarded as positive. Based on the intensity of color, uncolored, light yellow, yellowishbrown, and brown were scored as $0,1,2$, and 3 , respectively. The percentage of stained cells in the field of view was calculated as follows: five consecutive high-powered fields in each section were observed under a 400x optical microscope, and then the scores were averaged. The proportion of positive cells $<5 \%$ was recorded as $0,5 \%-25 \%$ as $1,26 \%-50 \%$ as 2 , $51 \%-75 \%$ as 3 , and $>75 \%$ as 4 . The final score was equal to the multiplication of the two scores: 0-2 as negative (-), 3-4 as weakly positive (+), and 5-12 as strongly positive $(2+/ 3+)$ expression [11].

\section{Statistical analysis}

Thehistopathologicalandimmunohistochemical data were then transferred to the SPSS Software program, version 25 to be statistically analyzed. Simple descriptive statistics (arithmetic mean and standard deviation) were used for the summary of quantitative data and frequencies were used for qualitative data. Estimation of the association between categorical variables was performed using the chi-square test. $p<0.05$ is considered as statistically significant.

\section{Results}

This study included forty cases of endometrial endometrioid carcinoma and thirty cases of endometrial hyperplasia. Fifty-seven percent (57\%) of studied cases were endometrial endometrioid carcinoma, $21.5 \%$ of cases were endometrial hyperplasia without atypia and $21.5 \%$ were atypical endometrial hyperplasia. The age of endometrial malignancy cases ranged in age from 39 to 77 years with a mean of age 62 years. Concerning FIGO grade of carcinoma cases, $35 \%$ of the cases were Grade 1, 37.5\% were Grade 2, and $27.5 \%$ were Grade 3. Regarding the FIGO stage, $55 \%$ of the cases were classified as FIGO Stage I, $27.5 \%$ of the cases were FIGO Stage II, and $17.5 \%$ of the cases were FIGO Stage III.

Less than half myometrial invasion was documented in $57.5 \%$ of the cases and more than half myometrial invasion was documented in $42.5 \%$ 
of the cases. Cervical, serosal, and/or adnexal and parametrial involvement by the tumor was detected in $27.5 \%, 10 \%$, and $7.5 \%$ of the cases, respectively. The pathological characteristics of the studied carcinoma cases are summarized in Table 1 [1].

Table 1: Pathological characteristics of the studied endometrial carcinoma cases

\begin{tabular}{lll}
\hline Pathological characteristics & & Number (\%) \\
\hline Histopathological type & Endometrioid carcinoma & $40(100)$ \\
FIGO grade & Grade 1 & $14(35)$ \\
& Grade 2 & $15(37.5)$ \\
FIGO Stage & Grade 3 & $11(27.5)$ \\
& I & $22(55)$ \\
Myometrial invasion & II & $11(27.5)$ \\
& LII & $7(17.5)$ \\
Cervical involvement & Less than half & $23(57.5)$ \\
& More than half & $17(42.5)$ \\
Serosal and/or adnexal involvement & Present & $11(27.5)$ \\
& Present & $29(72.5)$ \\
Parametrial involvement & Absent & $4(10)$ \\
& Present & $36(90)$ \\
& Absent & $3(7.5)$ \\
& & $37(92.5)$ \\
\hline
\end{tabular}

In the current study, 32 cases out of the 40 cases of endometrial endometrioid carcinoma (80\%) showed positive HE4 immunohistochemical expression in tumor cells while only 17 out of the 30 cases of endometrial hyperplasia (57\%) showed positive HE4 immunohistochemical expression. Seventeen cases of endometrial carcinoma (42.5\%) showed weak positivity for HE4immunohistochemical expression and 15 cases $(37.5 \%)$ showed strong HE4 positivity, while the remaining $20 \%$ of the cases were negative for HE4 immunohistochemical expression. Seventeen cases of endometrial hyperplasia (47\%) were weakly positive for HE4 immunohistochemical expression and only 3 cases (10\%) were strongly HE4 positive, while the remaining 13 cases (43\%) showed negative HE4 immunohistochemical expression (Table 2 and Figure 1).

Table 2: HE4 immunohistochemical expression in studied cases of endometrial endometrioid carcinoma and endometrial hyperplasia and its correlation with various pathological characteristics

\begin{tabular}{|c|c|c|c|c|}
\hline Parameters & $\begin{array}{l}\text { Negative } \\
(\%)\end{array}$ & $\begin{array}{l}\text { Weak } \\
\text { expression (\%) }\end{array}$ & $\begin{array}{l}\text { Strong } \\
\text { expression (\%) }\end{array}$ & Total (\%) \\
\hline Endometrial carcinoma & $8(20)$ & $17(42.5)$ & $15(37.5)$ & $40(100)$ \\
\hline Endometrial hyperplasia & $13(43)$ & $14(57)$ & $3(10)$ & $30(100)$ \\
\hline EH without atypia & $10(67)$ & $5((33)$ & $0(0)$ & $15(100)$ \\
\hline Atypical EH & $3(20)$ & $9(60)$ & $3(20)$ & $15(100)$ \\
\hline \multicolumn{5}{|l|}{ FIGO Grade } \\
\hline Grade I & $3(21)$ & $8(58)$ & $3(21)$ & $14(100)$ \\
\hline Grade II & $3(20)$ & $7(47)$ & $5(33)$ & $15(100)$ \\
\hline Grade III & $2(18)$ & $2(18)$ & $7(64)$ & $11(100)$ \\
\hline \multicolumn{5}{|l|}{ FIGO stage } \\
\hline Stage I & $4(18)$ & $12(55)$ & $6(27)$ & $22(100)$ \\
\hline Stage II & $2(18)$ & $5(46)$ & $4(36)$ & $11100 \%$ \\
\hline Stage III & $2(29)$ & $0(0)$ & $5(71)$ & $7(100)$ \\
\hline \multicolumn{5}{|l|}{ Myometrial invasion } \\
\hline $\begin{array}{l}\text { Less than half myometrial } \\
\text { thickness }\end{array}$ & $5(22)$ & $13(56)$ & $5(22)$ & $23(100)$ \\
\hline $\begin{array}{l}\text { More than half myometrial } \\
\text { thickness }\end{array}$ & $3(18)$ & $4(23)$ & $10(59)$ & $17(100)$ \\
\hline \multicolumn{5}{|l|}{ Cx involvement } \\
\hline Present & $2(18)$ & $5(46)$ & $4(36)$ & $11(100)$ \\
\hline Absent & $6(21)$ & $12(41)$ & $11(38)$ & $29 \%(100)$ \\
\hline \multicolumn{5}{|l|}{ Parametrial involvement } \\
\hline Present & $1(33)$ & $0(0)$ & $2(67)$ & $3(100)$ \\
\hline Absent & $7(19)$ & $17(46)$ & $13(35) \%$ & $37(100)$ \\
\hline \multicolumn{5}{|l|}{ Serosal/adnexal involvement } \\
\hline Present & $1(25)$ & $0(0)$ & $3(75)$ & $4(100)$ \\
\hline Absent & $7(20)$ & $17(47)$ & $12(33)$ & $36(100)$ \\
\hline
\end{tabular}

Endometrial endometrioid carcinoma cases showed higher percentage $(37 \%)$ of strongly positive cases for HE4 immunohistochemical expression than endometrial hyperplasia (only 10\%) with statistically significant relation ( $p=0.01$ ) (Table 2 and Figure 1).

Strong expression of HE4 was significantly higher in endometrioid carcinoma (37\%) than in endometrial hyperplasia without atypia which showed no strongly positive cases ( $p=0.005$ ) (Table 2 and Figure 1). There was no statistically significant relation in HE4 expression between endometrioid carcinoma and endometrial atypical hyperplasia $(p=0.06)$ (Table 2).

There was statistically significant relationship between the grade and the level of HE4 expression as Grade 3 carcinoma cases showed the highest percentage of strongly positive cases $(64 \%)$, followed by Grade $2(33 \%)$ followed by Grade $1(21 \%)(p=0.04)$ (Table 2, Figure 1).

Stage III endometrioid carcinoma cases showed the highest percentage of strongly positive cases $(71 \%)$, followed by stage II $(36 \%)$ followed by stage I $(27 \%)$ with statistically significant relation $(p=0.03)$ (Table 2, Figure 1).

Endometrial endometrioid carcinoma cases invading more than half myometrial thickness showed higher percentage of strongly positive cases (59\%), than those with less than half myometrial thickness with statistically significant relation $(p=0.02)$ (Table 2).

There was no statistically significant relation was detected in HE4 immunohistochemical expression among the endometrioid carcinoma cases with and without cervical involvement $(p>0.05)$. Furthermore, there was no statistically significant relation was detected in HE4 immunohistochemical expression among the endometrioid carcinoma cases with and without parametrial, serosal/adnexal involvement ( $p>0.05)$ (Table 2).

\section{Discussion}

Endometrial cancer is the most common gynecological malignancy. The available histopathological and clinical data do not allow for an efficient and well reproducible risk classification. This is especially true for an early-stage disease where few patients suffer fatal relapse in spite of the absence of the established high-risk criteria. Furthermore, no effective biomarkers currently exist to allow for an efficient risk classification of endometrial carcinoma, to direct treatment (chemotherapy and/or adjuvant radiation) in endometrial cancer, or to triage pelvic and para-aortic lymphadenectomy [19].

HE4 protein has gained a great degree of interest as a complementary biomarker to CA 125 , or even as an independent one for monitoring, diagnosis, and prognostic evaluation of ovarian cancer. They have 


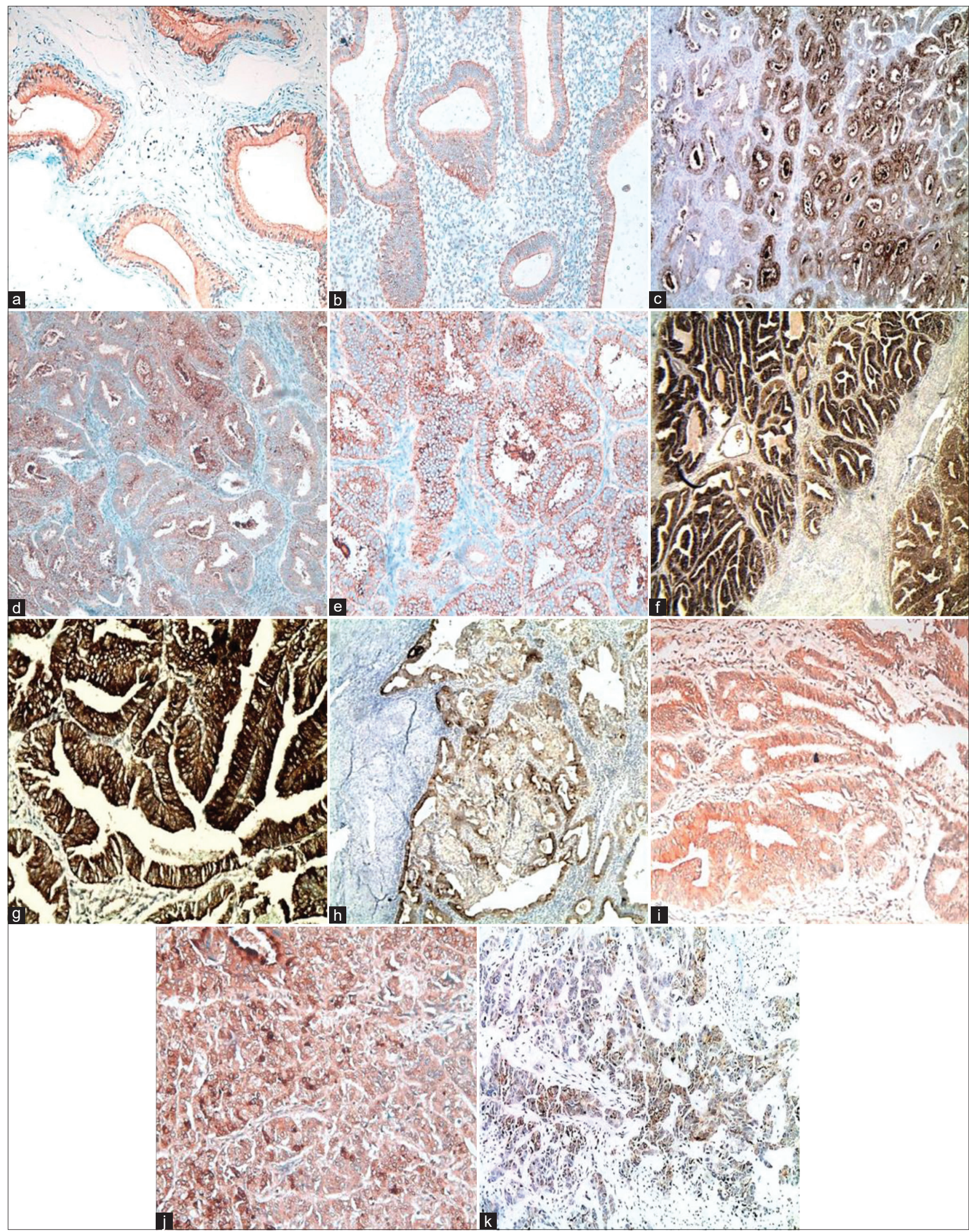

Figure 1: (a) Epidydimis (positive control) showing positive immunhistochemical expression of HE4. (b) Simple endometrial hyperplasia without atypia showing weekly positive immunhistochemical expression of HE4. (c) Complex atypical endometrial hyperplasia showing strongly positive immunohistochemical expression of HE4. (d) Complex atypical endometrial hyperplasia showing strongly positive immunohistochemical expression of HE4. (e) Complex atypical endometrial hyperplasia showing strongly positive immunohistochemical expression of HE4. (f) Grade 1 endometrioid carcinoma showing strongly positive immunohistochemical expression of HE4. (g) Villoglandular endometrioid carcinoma grade 1 showing strongly positive immunohistochemical expression of HE4. (h) Grade 2 endometrial endometrioid carcinoma showing weekly positive immunhistochemical expression of HE4. (i) Grade 2 endometrial endometrioid carcinoma showing strongly positive immunohistochemical expression of HE4. (j) Grade 3 endometrial endometrioid carcinoma showing strongly positive immunhistochemical expression of HE4. (k) Grade 3 endometrial endometrlioid carcinoma showing weekly positive immunhistochemical expression of HE4 
suggested that it could also be used in other types of cancers [20].

This study included forty cases of endometrial endometrioid carcinoma obtained from total hysterectomy with bilateral salpingoophrectomy and thirty cases of endometrial hyperplasia obtained from endometrial biopsies. All were obtained through collection of archived paraffin blocks during the period from January 2018 till February 2019, from the Pathology Department, Kasr Al-Ainy, Faculty of Medicine, Cairo University.

The mean age of studied endometrial endometrioid carcinoma cases was 62 years (ranging between 39-77 years). This is consistent to surveillance, epidemiology, and end result program (SEER), where the mean age at presentation for EC was reported to be 62 years [21] and was 57.6 years according to the pathology-based cancer registry of Ain-Shams Faculty of Medicine [22].

HE4 was mainly expressed in the cell membrane, and the cytoplasm also showed slight expression. In this study, the positive expression rate of HE4 was $80 \%$ in the endometrial cancer group, higher than $67 \%$ in the endometrial hyperplasia group, this figure close to that reported by Li et al., 2015 and Deng et al., 2015 [10], [11]. The positive expression rate of HE4 was $(84.62 \%$ and $85.7 \%$ ) in the endometrial cancer group higher than $(66.67 \%$ and $66.7 \%)$ in the endometrial hyperplasia group respectively. In accordance to our study, Yang et al. (2011) detected HE4 immunohistochemical expression in 31 cases of endometrial carcinoma, 19 cases of endometrial hyperplasia, they showed that the expression of HE4 in the malignant group was significantly higher than that in the hyperplasia group suggesting that HE4 may be involved in the tumor development [12].

In this work, the results showed that the strongly positive expression rate of HE4 in endometrioid carcinoma cases $(37.5 \%)$ was significantly higher than that in endometrial hyperplasia cases (10\%) and no cases of endometrial hyperplasia without atypia were strongly positive for HE4 $(0.0 \%)$, the figures are close to study reported by Li et al., 2015 which revealed that the strong expression of HE4 was significantly higher in endometrioid carcinoma $(55.98 \%)$ than that in endometrial hyperplasia (20\%) and also significantly higher than that in endometrial hyperplasia without atypia (0.0\%) [11]. Similarly, Deng et al., 2015 also reported that strong HE4 immunohistchemical expression is significantly higher in endometrial carcinoma cases $(45.2 \%)$ than that detected in endometrial hyperplasia cases (23.3\%) [12]. Zhang et al., 2016 stated that HE4 expression was obviously higher in patients with endometrial carcinoma than in benign uterine diseases [23].

In our study, there was statistically significant relationship between the grade and the level of HE4 expression as Grade 3 carcinoma cases showed the highest percentage of strongly positive cases (64\%), followed by Grade 2 (33\%) followed by Grade 1 (21\%) $(p=0.04)$. In agreement to our results, Li et al., 2015 reported that as the degree of endometrial cancer differentiation decreased, the HE4 level increased, and the HE4 positive expression rate in the poorly differentiated group (92.3\%) was significantly higher than that in the highly differentiated group (72.7\%), which demonstrated that HE4 expression in endometrial cancer is related to the degree of differentiation of the tumor [11]. Furthermore, Mutz-Dehbalaie et al., 2012, Bignotti et al., 2011; Moore et al., 2011 and Moore et al., 2008 reported positive correlation between FIGO grading and high expression of HE4 [9], [24], [25], [26].

In this work, FIGO Stage III cases showed the highest percentage of strongly positive cases $(71 \%)$, followed by stage II (36\%) followed by stage I $(27 \%)$ with statistically significant relation $(p=0.03)$. Similarly, Li et al., 2015 stated that the positive expression of HE4 in advanced endometrial cancer was significantly higher than that at earlier stages [11]. This is consistent with the findings in the literature of Li et al., 2013, Moore et al., 2011 and Bignotti et al., 2011, which suggested that the expression level of HE4 is associated with myometrial invasion depth; the larger the area and greater the depth of invasion, the more malignant cells are present and the higher the HE4 expression in corresponding tissues [25], [26], [27]. Furthermore, Deng et al., 2015 reported that the high expression rate of HE4 in Stage III-IV endometrial carcinoma was $66.7 \%(16 / 24)$, which was significantly higher than stage I-II 36.7\% (22/60) [10].

In our study, endometrial endometrioid carcinoma cases invading more than half myometrial thickness showed higher percentage of strongly positive cases $(59 \%)$, than those with less than half myometrial thickness with statistically significant relation. Similarly, Li et al. (2015), Li et al. (2013), Moore et al. (2011), and Bignotti et al. (2011) reported that the rate of HE4 positive expression increased with increasing the depth of myometrial invasion [9], [25], [26], [27]. In this study, there was no statistically significant relation was detected in HE4 immunohistochemical expression among the endometrioid carcinoma cases with and without cervical involvement. This correlation was not evaluated by other comparative studies. As well, no statistically significant relation was detected in HE4 immunohistochemical expression among the endometrioid carcinoma cases with and without serosal/adnexal or parametrial involvement. This might be explained because of the small number of cases within the groups of cervical, adnexal and parametrial involvement. These correlations were not evaluated by other comparative studies.

Finally, it is worth mentioning that one of the most important findings is that the positivity rate of HE4 expression was significantly higher in endometrial 
carcinoma than that in endometrial hyperplasia and also significantly higher than that in endometrial hyperplasia without atypia. This is consistent with many of the reported studies which provide a preliminary theoretical reference for basic research on the role of HE4 in the development of endometrial cancer.

Therefore, during interpretation of endometrial biopsies of atypical hyperplasia, HE4 strong expression might raise the possibility of the presence of coexisting adenocarcinoma not biopsied or even warning of a near future malignant transformation. Also, strong expression of HE4 by tissue biopsy of adenocarcinoma should be reported as this might predict higher grade and stage of the tumor, a point that should be considered by surgeons while performing hysterectomy. These results should be further confirmed by extending the study on a large scale, correlation of HE4 expression with the molecular classification of Tumor Cancer Genome Atlas and with patient's prognosis, particularly occurrence of recurrence and survival to establish the prognostic significance of HE4 expression in endometrial carcinoma and atypical hyperplasia.

\section{References}

1. Brennan DJ, Hackethal A, Metcalf AM, Coward J, Ferguson K, Oehler MK, et al. Serum HE4 as a prognostic marker in endometrial cancer-a population based study. Gynecol Oncol. 2014;132(1):159-65. https://doi.org/10.1016/j. ygyno.2013.10.036

PMid:24211402

2. Doll A, Abal M, Rigau M, Monge M, Gonzalez M, Demajo S, et al. Novel molecular profiles of endometrial cancer-new light through old windows. Mol Biol. 2008;108(3-5):221-9. https://doi. org/10.1016/j.jsbmb.2007.09.020

PMid:18061438

3. Jiang LN, Zhang HJ, Zhang HJ. The study of HE4, CA125 and CA72-4 for differential diagnosis between ovarian endometriosis and ovarian cancer. Chin J Lab Diagn. 2014;18(3):394-6.

4. Sandri MT, Bottari F, Franchi D, Boveri S, Candiani M, Ronzoni S, et al. Comparison of HE4, CA125 and ROMA algorithm in women with a pelvic mass: Correlation with pathological outcome. Gynecol Oncol. 2013;128(2):233-8. https://doi.org/10.1016/j. ygyno.2012.11.026

\section{PMid:23200911}

5. Schummer M, Ng WV, Bumgarner RE, Nelson PS, Schummer B, Bednarski DW, et al. Comparative hybridization of an array of 21, 500 ovarian cDNAs for the discovery of genes overexpressed in ovarian carcinomas. Gene. 1999;238(2):375-85. https://doi. org/10.1016/s0378-1119(99)00342-x

PMid:1057096

6. Yu S, Yang HJ, Xie SQ, Bao YX. Diagnostic value of HE4 for ovarian cancer: A meta-analysis. Clin Chem Lab Med. 2012;50(8):1439-46.

PMid:22868811

7. Karlsen NS, Karlsen MA, Hogdall CK, Hogdall EV. HE4 tissue expression and serum HE4 levels in healthy individuals and patients with benign or malignant tumors: A systematic review. Cancer Epidemiol Biomarkers Prev. 2014;23(11):2285-95. https://doi.org/10.1158/1055-9965.epi-14-0447

PMid:25169975

8. Galgano MT, Hampton GT, Frierson HF Jr. Comprehensive analysis of HE4 expression in normal and malignant human tissues. Mod Pathol. 2006;19(6):847-53. https://doi.org/10.1038/ modpathol.3800612

PMid:16607372

9. Moore RG, Brown AK, Miller MC, Badgwell D, Lu Z, Allard WJ, et al. Utility of a novel serum tumor biomarker HE4 in patients with endometrioid adenocarcinoma of the uterus. Gynecol Oncol. 2008;110(2):196-201. https://doi.org/10.1016/j. ygyno.2008.04.002

PMid:18495222

10. Deng L, Gao Y, Li X, Cai M, Wang H, Zhang H, et al. Expression and clinical significance of annexin A2 and human epididymis protein 4 in endometrial cancer. J Exp Clin Cancer Res. 2015;34(1):96. https://doi.org/10.1186/s13046-015-0208-8 PMid:26362938

11. Li J, Lin J, Luo Y, Kuang M, Liu Y. A multivariate analysis of prognostic biomarkers in surgically treated endometrial cancer. PLoS One. 2015;10(6):e0130640. https://doi.org/10.1371/ journal.pone. 0130640 PMid:26107255

12. Yang $\mathrm{CH}, \mathrm{Xu} Y \mathrm{YH}$, Zhang $\mathrm{XY}$. Expression and significance of human epididymis gene product 4 in tissue and serum of patients with endometrial cancer. Chin J Clin Oncol. 2011;38(9):516-9.

13. Lu R, Sun X, Xiao R, Zhou L, Gao X, Guo L. Human epididymis protein 4 (HE4) plays a key role in ovarian cancer cell adhesion and motility. Biochem Biophys Res Commun. 2012;419(2):27480. https://doi.org/10.1016/j.bbrc.2012.02.008 PMid:22342977

14. Trudel D, Tetu B, Gregoire J, Plante M, Renaud MC Bachvarov D, et al. Human epididymis protein 4 (HE4) and ovarian cancer prognosis. Gynecol Oncol. 2012;127(3):511-5. https://doi.org/10.1016/j.ygyno.2012.09.003 PMid:22967799

15. Zaino R, Carinelli SG, Ellenson LH. Epithelial tumors and precursors. In: Kurman RJ, editor. WHO Classification of Tumours of the Female Reproductive Organs. Lyon, France: IARC; 2014. p. 125-35.

16. Zhou Q, Singh SR, Yunzhe L, Mo Z, Huang J. Preoperative histopathological grading and clinical staging versus surgicopathological grading and surgical staging in endometrial carcinoma patients: A single centre retrospective study. Glob J Med Res. 2018;18:18-28.

17. FIGO Committee on Gynecologic Oncology. FIGO staging for carcinoma of the vulva, cervix, and corpus uteri. Int $J$ Gynaecol Obstet. 2014;125(2):97-8. https://doi.org/10.1016/j. ijgo.2014.02.003 PMid:24630859

18. Powéll MA, Olawaiye $A B$, Bermudez A. Corpus uteri-carcinoma and carcinosarcoma. In: Amin MB, Edge SB, Greene FL, editors. AJCC Cancer Staging Manual. $8^{\text {th }}$ ed. New York: Springer; 2017. p. 639-57.

19. Kommoss F, Grevenkamp F, Bunz AK, Taran FA, Fend F, Lax S. L1CAM: Amending the low-risk category in endometrial carcinoma. J Cancer Res Clin Oncol. 2017;143(2):255-62. https://doi.org/10.1007/s00432-016-2276-3 PMid:27695947

20. Kumarasamy C, Madhav MR, Sabarimurugan S, Lakhotiya K, Pandey V, Priyadharshini T, et al. Diagnostic and prognostic role of HE4 expression in multiple carcinomas: A protocol for systematic review and meta-analysis. Medicine (Baltimore). 2019;98(28):e15336. https://doi.org/10.1097/ md. 0000000000015336 
PMid:31305389

21. Khatuja R, Rai S. Endometrial carcinoma: Epidemiology and risk factors. In: Preventive Oncology for the Gynecologist. Singapore: Springer; 2019. p. 3-12. https://doi. org/10.1007/978-981-13-3438-2_1

22. Helal EA, Salman MI, Ezz-Elarab SS. Malignant tumors of the body of uterus. In: Pathology-Based Cancer Registry 20022010. $1^{\text {st }}$ ed. Cairo, Egypt: Ain-Shams Faculty of Medicine; 2015. p. 54-56

23. Zhang $Z$, Chen $H X, X u X X$. The values of the combined detection of serum human epididymis protein E4, CA125 and CA199 in diagnosis of endometrial cancer. J Pract Med. 2016;32(7):1108-12.

24. Mutz-Dehbalaie I, Egle D, Fessler S, Hubalek M, Fiegl H, Marth $\mathrm{C}$, et al. HE4 is an independent prognostic marker in endometrial cancer patients. Gynecol Oncol. 2012;126(2):18691. https://doi.org/10.1016/j.ygyno.2012.04.022

\section{PMid:22525819}

25. Bignotti E, Ragnoli M, Zanotti L, Calza S, Falchetti M Lonardi S, et al. Diagnostic and prognostic impact of serum HE4 detection in endometrial carcinoma patients. Br J Cancer. 2011;104(9):1418-25. https://doi.org/10.1038/ bjc. 2011.109

PMid:21468050

26. Moore RG, Miller MC, Disilvestro P, Landrum LM, Gajewski W, Ball $\mathrm{JJ}$, et al. Evaluation of the diagnostic accuracy of the risk of ovarian malignancy algorithm in women with a pelvic mass. Obstet Gynecol. 2011;118(2):280-8. https://doi.org/10.1097/ aog.0b013e318224fce2

27. Li J, Chen H, Mariani A, Chen D, Klatt E, Podratz K, et al. HE4 (WFDC2) promotes tumor growth in endometrial cancer cell lines. Int J Mol Sci. 2013;14(3):6026-43. https://doi.org/10.3390/ ijms14036026

PMid:23502467 